\title{
TASTE AND SINGULAR ECONOMIES
}

\author{
Elvio Accinelli *
}

Facultad de Ingeniería, Instituto de Matemáticas y Estadistica Rafael Laguardia, Universidad de la República, Montevideo, Uruguay

\section{Alfredo Piria}

Facultad de Ingeniería, Instituto de Matemáticas y Estadística Rafael Laguardia, Universidad de la República, Montevideo, Uruguay

\section{Martín Puchet}

Facultad de Economía, Universidad Nacional Autónoma de México

(Received 14 November 2002, accepted 6 February 2003)

\begin{abstract}
The purpose of this paper is to show some examples, and characteristics of the economies where, as a consequence of changes in the utility functions, the set of equilibria changes. These changes play a crucial role to understand the behavior of the economy as a system. An economy will be called singular if small changes in the tastes of the consumers imply big changes in the set of equilibria. In this paper, we use the Negishi (1960) approach to model the economic system composed by a finite number of agents and goods, to do so we use the concepts of Morse and stable functions to characterize the singular economies.

\section{Resumen}

El propósito de este trabajo es mostrar algunas caracteristicas y ejemplos de las economías donde, a consecuencia de cambios en las funciones de utilidad, el conjunto de equilibrio cambia. Estos cambios juegan un papel crucial para entender el comportamiento de la economia como un sistema. Se dice que una economía es singular si pequeños cambios en las preferencias de los consumidores generan grandes cambios en el conjunto de equilibrio. En este trabajo se utiliza la aproximación de Negishi (1960) para modelar un sistema económico con un número finito de agentes y bienes, para lo cual se utilizan los conceptos de Morse y funciones estables con el fin de representar a las economías singulares.
\end{abstract}

JEL classification: D5, E21

Keywords: General equilibrium, Consumption

* Instituto de Matemáticas y Estadística Rafael Laguardia, Facultad de Ingeniería. Universidad de la República. Julio Herrera y Reissig, no. 565, C.P. 11300, Telephone (598)(2) 7110621, Montevideo, Uruguay. E-mail: elvio@fing.edu.uy

The authors are grateful to Yves Balasko for helpful comments. The commentator is not responsible for any persisting errors. 


\section{Introduction}

The object of this paper is to show some examples of pure exchange economies, where small changes in the tastes of the agents imply large changes in the behavior of the economic system. These kind of economies are called singular economies. In our work, the tastes of the agents are modelled by parametrized utility functions. Following the Negishi (1960) approach, we will characterize each economy by its excess utility function. Moreover, in this paper, each parametrized economy is characterized by a parametrized excess utility function. It is then possible to distinguish among economies by observing the different excess utility functions defined by different values of the parameters. Rigorously speaking, an economy is singular if in every one of its neighborhoods there exists another economy whose behavior is substantially different with respect to the behavior of the previously considered, this means that, in every neighborhood of the excess utility function that corresponds to the former economy, there exists another excess utility function that corresponds to another economy, such that this function displays a different topological behavior. Traditionally, the study of the singularities is made considering the utilities as given and endowments as parameters, see the pioneer article of Balasko (1978). In our approach, we consider, firstly, endowments as fixed, and we analyze the changes in the behavior of the economies when the utility functions change.

In the traditional approach, it is well known that in a neighborhood of a singular economy, other economies with different number of Walrasian equilibria can be found, see Accinelli (1996). This means that if utilities are fixed, in every neighborhoods of a certain endowment there will exist another endowment such that for the given utilities, the number of equilibria is different. We will see that this is also the behavior of a singular economy when we consider utilities as variables, and endowments fixed.

The use of the Negishi approach is determined by the object of this paper. In this approach the characterization of the Walrasian equilibrium set is given by the excess utility function. This function plays a fundamental role in our work because:

i) The utilities appear explicitly in the excess utility function

ii) The zeroes of this function are in one to one correspondence with the Walrasian equilibria.

Then, changes in the utility functions appear to be directly related with changes in the Walrasian equilibrium set. In our approach, the singularity appears as a property of the utilities of consumers. This means that, the tastes of the consumers play crucial role when characterizing a singular economy.

We will consider the initial endowments as given, and we will show cases in which in a neighborhood of a given excess utility function, economies with a different number of equilibria exist. This means that, if the economy is singular in this sense, a little modification in the utilities of the consumers, or little mistakes in the measure or appreciation of their tastes, may give rise to an unforeseen behavior. The mathematical statement of this phenomenon is that in a neighborhood of a singular economy, represented by a specific excess utility function, a small perturbation of the parameters leads the system to different 
equilibria sets which are structurally diverse. To deal with this type of changes in the metric space of the functions, we introduce the topology of the uniform convergence, and thereafter apply Morse functions to classify it as regular or singular economies, depending on its corresponding excess utility functions.

Note that all considerations that we will do in terms of the excess utility functions could be done in terms of the excess demand function. In the same way, we will use in this paper Morse theory, and the excess utility function to characterize the set of the two-agents, and $l$-goods economies; using Morse theory, and the excess demand function it is possible to characterize $n$-agents, and 2-goods economies.

The order of the sections is as follows. Section 2 presents the definitions of regular and singular economies in the Negishi approach, and shows the principal economic consequences of the use of this approach. Section 3 contains examples of the effects of parametric changes in the behavior of the economic system. In section 4, the space function with topology of the uniform convergence, and the Morse functions are defined, and then used to classify economies. Here, we also introduce additional examples. The last section concludes the economic meaning of the relationship between the tastes of the consumers, and the behavior of the economic system. Some appendix appear at the end of the paper.

\section{Regular and Singular Economies in the Negishi Approach}

A pure exchange economic system is characterized by the set $\varepsilon=\left\{u_{i}, w_{i}, I\right\}$, where preferences are represented by the utility functions $u_{i}: \mathbb{R}_{+}^{l} \rightarrow \mathbb{R}, \forall i \in I$, endowments of the agent $i$ are denoted by the symbol $w_{i}$, and $I$ is a finite index set. In our work we are assuming that preferences can be represented by utility functions, and $X=\mathbb{R}_{+}^{l}$ is the consumption space of the agent $i$. Therefore we will deal only with economies with a finite number of agents, and goods.

The social welfare function of an economy; $W_{\lambda}: \mathbb{R}_{+}^{n l} \rightarrow \mathbb{R}$ defined as:

$$
W_{\lambda}(x)=\sum_{i=1}^{n} \lambda_{i} u_{i}\left(x_{i}\right),
$$

where $u_{i}$ is the utility function of the agent indexed with $i$, and

$$
\lambda=\left(\lambda_{1}, \lambda_{2}, \ldots, \lambda_{n}\right) \in \Delta_{+}=\left\{\lambda \in \mathbb{R}_{++}^{n}: \sum_{i=1}^{n} \lambda_{i}=1\right\} .
$$

Each $\lambda_{i}$ represents the social weight of the agent $i$ in the market.

As it is well known, if $x^{*} \in \mathbb{R}^{n l}$ solves the maximization problem of $W_{\lambda^{*}}(x)$ subject to being a feasible allocation, i.e.,

$$
x^{*} \in \mathcal{F}=\left\{x \in \mathbb{R}_{+}^{n l}: \sum_{i=1}^{n} x_{i} \leq \sum_{i=1}^{n} w_{i}\right\},
$$


then $x^{*}$ is a Pareto optimal allocation (sec Mas-Colell, Whinston, and Green, 1995). Reciprocally it can be proven that if a feasible allocation is Pareto optimal, then there exists a $\lambda^{*} \in \Delta$

$$
\Delta=\left\{\lambda \in \mathbb{R}_{+}^{n}: \sum_{i=1}^{n} \lambda_{i}=1\right\}
$$

such that $x^{*}$ maximizes $W_{\lambda^{*}}$.

If we are to consider every Pareto optimal allocation, we will need to consider cases where $\lambda_{j}=0$ for some $j \in\{1,2, \ldots, n\}$. In these cases the agents indexed in this subset will be out of the market. As utilities are strictly increasing, the maximization process implies that this agent will receive $x_{j}=0$. Since we consider each agent to have a non-null endowment, this cannot be an equilibrium allocation. Then we can restrict ourselves, without loss of generality, to consider only cases where $\lambda \in \Delta_{++}$.

Once the set of Pareto optimal allocations has been characterized, our next step is to choose the elements $x^{*}$ in the Pareto optimal set that can be supported by a price $p$, satisfying the condition for an equilibrium allocation $p x^{*}=p w_{i}$, for all $i=1,2, \ldots, n$.

Definition 1: Let $e_{i, u_{i}, w_{i}}: \Delta_{+} \rightarrow \mathbb{R}, i=1,2, \ldots, n$ be the function

$$
e_{i, u_{i}, w_{i}}(\lambda)=\sum_{j=1}^{l} \frac{\partial u_{i}\left(x^{*}(\lambda)\right)}{\partial x_{i j}}\left(x_{i j}^{*}(\lambda)-w_{i j}\right),
$$

is the excess utility function for the agent $i, u_{i}$ is his utility function, and $w_{i j}$ is the endowment of this agent in the commodity $j$. The bundle set $x_{i}^{*}(\lambda)$ is risen from $x^{*}(\lambda)$ that maximizes $W_{\lambda}(x)$, s.t. : $\mathcal{F}$, with $i=1,2, \ldots, n$, and $j=1,2, \ldots, l$.

Definition 2: The excess utility function, $e_{u_{1}, u_{2}, \ldots u_{n}, w}: \Delta_{+} \rightarrow \mathbb{R}^{n}$ is the vector

$$
e_{u, w}(\lambda)=\left(e_{1, u_{1}, w_{1}}(\lambda), e_{2, u_{2}, w_{2}}(\lambda), \ldots, e_{n, u_{n}, w_{n}}(\lambda)\right) .
$$

Assuming conditions such that the solution of the maximization program, involving the social welfare function, will be attained in the interior of $\mathbb{R}_{-}^{l}$, it follows that if $x^{*}(\lambda, w)$ is the allocation that solves this problem, we have

$$
\frac{\partial u_{i}\left(x^{*}(\lambda, w)\right)}{\partial x_{i j}}=\frac{1}{\lambda_{i}} \gamma_{j}(\lambda),
$$

and then

$$
e_{i, u_{i}, w_{i}}(\lambda)=\frac{1}{\lambda_{i}} \sum_{j=1}^{l} \gamma_{j}(\lambda)\left(x_{i j}^{*}(\lambda)-w_{i j}\right) .
$$

Let $\Omega=\Pi_{i=1}^{n} \mathbb{R}_{+}^{l}$ be the consumption space. 
Definition 3: For fixed utility functions $u$, and for each $w \in \Omega$ one can define the set

$$
\varepsilon q(u, w)=\left\{\lambda \in \Delta_{+}: e_{u, w}(\lambda)=0\right\},
$$

that it is called the set of the Equilibrium Social Weights.

In Accinelli (1999), it is proven that it is a non-cmpty set. The following thcorem summarizes the main result of this approach applied to an economic system with a finite number of agents and goods.

Theorem 1: Let $\lambda \in \varepsilon q(u, w)$ be, and let $x^{*}(\lambda)$ be a feasible allocation, solution of the maximization problem of $W_{\lambda}$, and let $\gamma(\lambda)$ be the corresponding vector of Lagrange multipliers. Then, the pair $\left(x^{*}(\lambda), \gamma(\lambda)\right)$ is a Walrasian equilibrium, and reciprocally if $(p, x)$ is a Walrasian equilibrium then there exists $\bar{\lambda} \in \varepsilon q$ such that $x$ maximizes $W_{\bar{\lambda}}$ restricted to the feasible allocations set, and $p$ will be the corresponding vector of Lagrange multipliers, i.e., $p=\gamma(\bar{\lambda})$. (Accinelli, 1999).

The allocation $x(\lambda)$ is an equilibrium allocation if the excess utility function of cach agent vanishes at $x(\lambda)$. This means that $\lambda$ is in the equilibrium set $\varepsilon q(w)$, if and only if produces an allocation such that the gradient $\nabla u_{i}(x(\lambda))=$ $\left(\partial u_{i}(x(\lambda)) / \partial x_{1}, \partial u_{i}(x(\lambda)) / \partial x_{2}, \cdots, \partial u_{i}(x(\lambda)) / \partial x_{l}\right)$ of each utility $u_{i}$ cvaluated in $x_{i}(\lambda)$ is orthogonal to the bundle set $\left(x_{i}(\lambda)-w_{i}\right)$, for cach $i \in I$.

In this frame, it is possible to define:

Definition 4: A Singular Economy means that for fixed utility functions, and given endowments, there exist at least one set of social weights where the excess utility function vanishes: $\lambda \in \Delta$ where $e_{u, w}(\lambda)=0$, and rank $J e_{u, w}(\lambda)<n-1$. $\lambda$ is the vector of social weights, $e_{u, w}$ is the excess utility function, and $J e_{u, w}(\lambda)$ denotes the jacobian of this function, (Accinelli, 1999).

When $\operatorname{rank} J e_{u, w}(\lambda)=n-1$, we are in presence of a Regular Economy.

\section{Examples of Singular and Regular Economies}

Consider the following economic system composed of two agents and two goods:

$$
\begin{gathered}
u_{\alpha, 1}\left(x_{11}, x_{12}\right)=x_{11}-\frac{1}{\alpha} x_{12}^{-\alpha}, \\
u_{\alpha, 2}\left(x_{21}, x_{22}\right)=-\frac{i}{\alpha} x_{21}^{-\alpha}+x_{22},
\end{gathered}
$$

and the initial endowments: $w_{1}=\left(w_{11}, w_{12}\right), w_{2}=\left(w_{21}, w_{22}\right)$, for consumer 1 , and 2 respectively (this example, with $\alpha=8$ is given in Mas-Colell, Whinston, and Grecn, 1995).

The excess utility function of this system (see the Appendix) is:

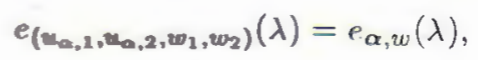


when $\alpha=12$, we obtain for $w_{1}=(2, r)$, and $w_{2}=(r, 2)$ with $r=2^{\frac{8}{9}}-2^{\frac{1}{9}}$, an economy with three relative weights of equilibrium $\lambda=(0.05,0.95) ;(0.5,0.5)$; $(0.95,0.5)$, that is, three different values of $\lambda$ such that $e_{\alpha, w}(\lambda)=0$.

If we consider $\alpha$ in an $\epsilon$-neighborhood of 12 , that is $\alpha \in(12-\epsilon, 12+\epsilon)$ the economy has the same behavior as in $\alpha=12$. That is, that the economy has three Walrasian equilibrium if utilities are chosen in this neighborhood. This is illustrated in figure 1.a.

If we assume $\alpha=5$, we obtain only one equilibrium with $\lambda=(0.5,0.5)$, and we will not observe significant changes due to little changes of this value of $\alpha$. But if $\alpha^{*}=7.76$, and we consider an $\epsilon$-neighborhood of $\alpha^{*}$ for fixed endowments $w$, then there exist different values of $\alpha$ where the excess utility function $e_{\alpha, w}(\lambda)$ has a different number of zeros. For this value $\alpha^{*}$, we say that the utility functions generate a singular economy, because in a neighborhood of this function there exist utility functions such that the respective excess utility function has a different number of zeros, and then the economy is structurally unstable in the sense that, a small change in the tastes of one consumer implies a qualitative, and quantitative change in the structure of the Walrasian equilibrium set. The cardinality of the equilibrium set changes from one to three, or reciprocally.

The first case is a regular economy that is obtained from a type of utility functions, and in the second case the economy is singular because the consumers have another type of utility functions. Then, in the first case, some changes in tastes, or mistakes in the consideration of utilities do not imply big differences in the expected behavior of the economy. Whereas in the second case, little mistakes in the measurement of the utilities of the agents, or small changes in tastes have implicit unpredictable consequences.

Figure 1.b shows changes in the equilibrium set when the parameter $\alpha$ changes, the bifurcation point is $\alpha=7.76$, for smaller values we can observe only one equilibrium $\lambda \rightleftharpoons(0.5,0.5)$, whereas beyond this point two new equilibrium branches appear. If we consider an economy with the same kind of utility functions as in the example, and the endowments $w_{11}=2 ; w_{12}=0.75 ; w_{21}=r$; $w_{22}=2$, we obtain the possibility of more sudden changes. Figure 2.a shows the equilibrium values of this economy, and figure 2.b shows the bifurcation point $\alpha^{*}=8.81$. If $\alpha \geq \alpha^{*}$ we observe that the economy has three branches, but when $\alpha<\alpha^{*}$, two of these branches disappear, and we obtain only one equilibrium value for $\lambda$, and only one equilibrium branch, see figure 4 . The possibility of sudden, and big changes in the equilibrium set is clear for economies in a neighborhood of this value. As all measures imply mistakes, the behavior of this kind of economies is absolutely unpredictable.

\section{The Metric Space of the Excess Utility Functions, and the Morse Functions}

In our work it is essential to have a notion of closeness for utility functions. Following Mas-Colell (1985), we will consider the topological space of functions $C^{r}(X)$, where $r \geq 1$ specifies that for every $0 \leq s \leq r$, the $s$ th-derivative of $f \in C^{r}(X)$ is continuous on $X$. The topology considered will be the topology of the $C^{r}$ uniform convergence. Notice that $f_{n} \rightarrow f$, if and only if every derivative of $f_{n} \rightarrow f$ up to the $r$ th order converges uniformly to zero. 
In this function space the following properties hold:

a) Every $C^{r}(X), 0 \leq r \leq \infty$, is metrizable, separable, and complete.

b) In the following sequence,

$$
C^{\infty}(X) \subset \ldots \subset C^{2}(X) \subset C^{1}(X) \subset C^{0}(X),
$$

the topologies are increasingly finer, and every space is more dense than the preceding one. Recall that $C^{\infty}(X, \mathbb{R})$ denotes the set of real valuefunctions $f: X \rightarrow \mathbb{R}$, whose derivatives of any order are continuous.

c) If $X$ is not compact, we give to $C^{r}(X)$ the topology of the $C^{r}$ uniform convergence on compact, that is, $f_{n} \rightarrow f$, if and only if $f_{n} / Y \rightarrow f / Y$ for every compact $Y \subset X$, in the previous sense.

These are well known properties of this space (see for instance Royden, 1963).

As we focus in utilities, and the implications that changes in utilities have on the economy regarding the number of equilibria, abruptness of the change of equilibria, etcetera; we will then deal with the relationship between the kind of excess utility functions, and their zeros. We will say that, an economy $\varepsilon^{\prime}$ is in a $\delta$-neighborhood, $\mathcal{U}_{\delta}$, of an economy $\varepsilon$, if the corresponding utilities $u_{\varepsilon}$, and $u_{\varepsilon^{\prime}}$ satisfy $\left\|u_{\varepsilon}-u_{\varepsilon^{\prime}}\right\|<\delta$, with the $C^{r}$ uniform topology. It follows that the economies $\varepsilon$, and $\varepsilon^{\prime}$ are in a $\mathcal{U}_{\delta}$, with $C^{r}$ uniform topology, if and only if in a neighborhood of an equilibrium, the respective excess utility functions $e$, and $e^{\prime}$ are in a $\delta$-neighborhood: $\left\|e-e^{\prime}\right\|<\delta$, with the $C^{r-1}$ uniform topology. We can say that a utility function for given endowments $w$ is singular, if in a neighborhood of this function, considering the compact uniform convergence, there exist utility functions such that the respective excess utility functions have a different number of zeros, i.e., a different number of Walrasian equilibria.

Figure 1.a.

Figure 1.b.
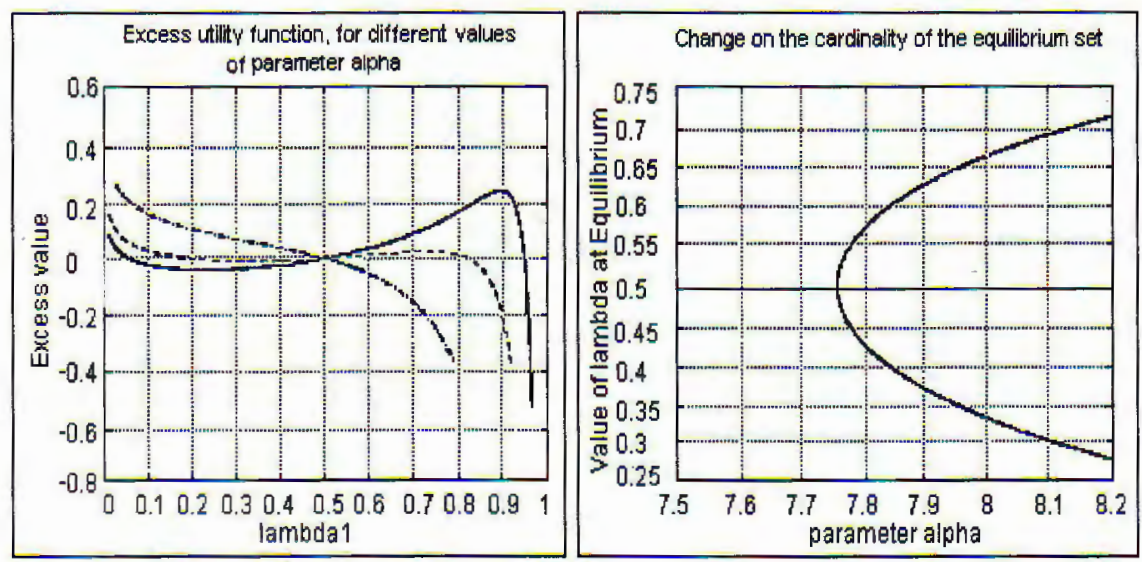

On the left, changes in utility, given by small changes in $\alpha$, imply changes in the number of the equilibria. On the right, the bifurcation of the equilibria; before the bifurcation point, economies have just one equilibrium; after $\alpha^{*}$, economies have three equilibria. 
Figure 2.a.

Figure 2.b.
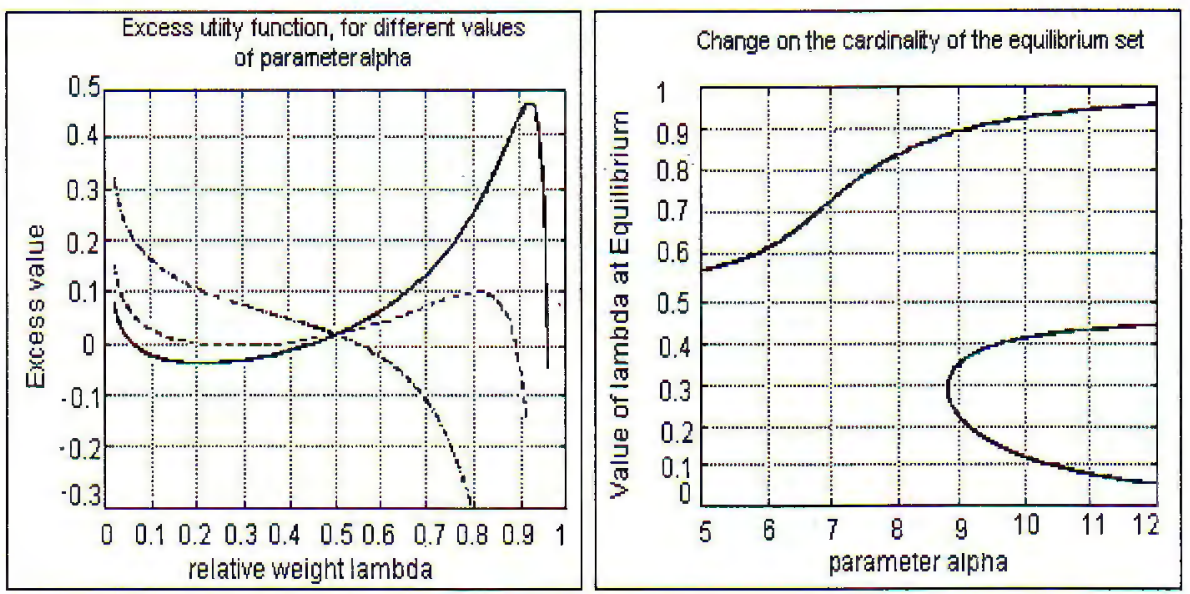

Another possible kind of bifurcation, the figure on the left shows a dramatic situation.

As the excess utility function satisfies the Walras law, $\lambda e_{u, w}(\lambda)=0$, and as we can consider $\sum_{i=1}^{n} \lambda_{i}=1$ (sec Accinelli, 1996), then in the case of an economy with two agents, to obtain the set of points $\lambda=\left(\lambda_{1}, \lambda_{2}\right)$, such that $e_{u, w}(\lambda)=0$ is enough to solve only one of the two equations $e_{i, u, w}\left(\lambda_{i}\right)=0$ as a function just one variable $\lambda_{i}, i=1,2$. If we assume that each agent has a positive endowment, and utilities are increasing, then it is possible to find a value $\epsilon>0$ such that all $\lambda_{i}$ in the cquilibrium set verify $\lambda_{i} \geq \epsilon$. So, in this case, the problem of solving the equilibrium is equivalent to obtaining the zeros of the function $e_{i, u, w}: X \rightarrow \mathbb{R}$, where $X=[\epsilon, 1]$.

We need the following definition:

Definition 5: Let $f$ be in $C^{\infty}(X, \mathbb{R})$, a function $f$ is a Morse function if all of the critical points of $f$ are non degenerates.

Recall that if $f: X \rightarrow \mathbb{R}$ is a smooth map, a point $p \in x$ is a critical point if $f^{\prime}(p): X \rightarrow \mathbb{R}$ is not onto. It is a well known fact that the set of Morse functions is a residual set in $C^{\infty}(X, \mathbb{R})$ (see Golubitsky, and Guillemin, 1973).

Definition 6: $f$, and $e$ in $C^{\infty}\left(X, \mathbb{R}^{n-1}\right)$ are equivalent if there exist diffeomorphisms $g: X \rightarrow X$, and $h: \mathbb{R}^{n-1} \rightarrow \mathbb{R}^{n-1}$, such that $f=h e g^{-1}$, and $f$ is stable if there is a neighborhood $W_{f}$ (in the sense of the uniform topology above considered) of $f$ in $C^{\infty}\left(X, \mathbb{R}^{n-1}\right)$ such that all $e \in W_{f}$ are equivalent to $f$.

We will say that an economy is stable, if the excess utility function is a stable map in the sense of Golubitsky, and Guillemin (1973) indicated above. So, if an cconomy is stable, the grafic representation of its excess utility function is similar to the excess utilities of the economies in which the tastes of the consumers are similar. The tastes of the agents are similar if the utilities that represent these tastes are in $\delta$-neighborhood. 
The following theorem is helpful in characterizing the behavior of the economies in a neighborhood of a singular economy, when we assume that an economy $\varepsilon^{\prime}$ is in the neighborhood of an economy $\varepsilon$, if and only if the cxcess utility function $e^{\prime}$ of $\varepsilon^{\prime}$ is in a ncighborhood of the excess utility function $e$ of $\varepsilon$.

From the mathematical point of view, the theorem sets that the stability in the function space with uniform convergence topology is equivalent to the Morse functions characterized by the fact that its critical points have separated images.

Theorem 2: Let $f$ be in $C^{\infty}(X, \mathbb{R})$ where $X$ is a compact manifold. Then $f$ is stable if and only if it is a Morse function where the critical values are distinct (i.e., if $x_{1}$, and $x_{2}$ are distinct critical points of $f$ in $X$, then $f\left(x_{1}\right) \neq f\left(x_{2}\right)$ ).

This means that, generically, the set of singular economies have one and only one singular equilibrium, and this singularity is a fold. The proof of this theorem can be found in Golubitsky, and Guillemin (1973).

On the other hand, it is a well known fact in Morse theory that the set of functions whose singularities are non degenerates is an open and dense set, and non degenerate critical points are isolated. Thus, generically, singular economies are stable.

\subsection{Example of Morse Excess Utility Function, and Singular Economy}

The example below shows a two consumers economy, whose excess utility function represcuts a singular, and regular economy. This is a singular economy, i.e., in all neighborhood of its cxcess utility function there are economies with a different number of equilibria.

Therefore, figures 1.a, and 2.a show a generic casc of a two agent economy. The excess utility function of each agent is a Morse function (sce Appendix), these functions have Jacobian of complete rank, and if they could have critical equilibrium points, these points would have different values which are not degenerates. This means that a singular economy will have only one singular, and no degenerate equilibrium. That is, if utilities, and endowments are given, there will be generically only one set of social weight, $\bar{\lambda}=\left(\bar{\lambda}_{1}, \bar{\lambda}_{2}\right)$ such that $e_{i, \alpha, w}\left(\bar{\lambda}_{i}\right)=0$, and $\partial e_{i, \alpha, w}\left(\bar{\lambda}_{i}\right) / \partial \lambda_{i}=0$. On the other hand, in any neighborhood of an excess utility function, $e_{i, \alpha, w}$, that is a Morse function, corresponding to a singular economy, there exist excess utility functions, $e_{i, \alpha^{\prime}, w}$, such that for each one of them, the primage of zero has three different points (each one of these three points is a regular equilibria for a regular cconomy, whose excess utility function is $\left.e_{i, \alpha^{\prime}, w}\right)$.

Additionally, excess utility functions, $e_{i, \bar{\alpha}, w}$, that vanish at only one point, corresponding to a regular economy with only one regular equilibrium, and whose cxcess utility function is $e_{i, \bar{\alpha}, w}$. To see this, it is enough to consider small modifications in the value of the parameter $\alpha$.

Recall that in the two consumers case it is enough to consider only one coordinate of the excess utility function. If the excess utility function $e:[0,1] \rightarrow \mathbb{R}$ of the cconomy $\varepsilon$ is a Morse function, all of whose critical values are distinct, then this excess utility function belongs to a residual set in $C^{\infty}(X, \mathbb{R})$. Moreover from the theorem above, it follows that, this excess utility is a stable function in the sense that, there is a neighborhood $W_{e}$ of e, such that all 
functions $e^{\prime} \in C^{\infty}(X, \mathbb{R})$ in this neighborhood will be equivalent to $e$. In spite of this, the singular equilibrium can disappear if there occurs a change in the tastes of the consumer, then all economy $\varepsilon^{\prime}$ in a neighborhood of $\varepsilon$ will have equivalent excess utility functions.

If the economy is singular, and the excess utility function has more than one singular equilibrium, that is if there are more than one critical point, $\lambda_{i}^{1} \neq$ $\lambda_{i}^{2}$, where $e\left(\lambda_{i}^{1}\right)=e\left(\lambda_{i}^{2}\right)=0$, then there will not be a stable economy, that means that we can not say anything about the behavior of the economies in a neighborhood of the singular economy.

The stable functions in $C^{\infty}(X, \mathbb{R})$ have a non-degenerate form since these are just the classical Morse functions. Such functions can only have one type of singularities (i.e., have only non-degenerate critical points, see Golubitsky, and Guillemin, 1973). Stable maps are also dense in $C^{\infty}(X, \mathbb{R})$, this property is also verified for functions in $C^{\infty}(X, Y)$, if $X$ and $Y$ are manifolds such that $\operatorname{dim} X=$ $\operatorname{dim} Y=2$, but unfortunately this is not the case if we consider $C^{\infty}(X, Y)$ for an arbitrary manifold $Y$ (see Accinelli, and Puchet, 2001). The general answer depends on the relative dimensions of $X$ and $Y$. This means that, in some cases there can exist one generic economy $\varepsilon^{\prime}$, such that in all neighborhoods $W_{\varepsilon^{\prime}}$ of $\varepsilon^{\prime}$, there exists an economy $\varepsilon$, such that has a non-equivalent behavior, i.e., the respective utility functions are not equivalent. Such posibility depends on the relative dimensions of the consumption space $X$, and the number of agents in the economy.

However if the economy is regular (i.e., the excess utility function $e$ : $\mathbb{R}^{n-1} \rightarrow \mathbb{R}^{n-1}$ is an immersion one to one) then $e$ is a stable map. We will finish this section by indicating the existence of certain dual behavior in the singular economies. To see this let us consider a parametrized excess utility function $e: \mathbb{R} \times, \Delta_{+}, \times \Omega \rightarrow \mathbb{R}^{n-1}$, where $\Delta_{+}$is the simplex, $\Omega$ is the consumption space, and $n$ is the number of agents in the economy. Let $e(\alpha, \lambda, w)$ be the excess utility function of $\varepsilon$, then, if the economy is singular in the sense of the utility functions, this means that if endowments $w \in \Omega$ are fixed, and small changes in tastes imply changes in the number of equilibria, then the economy is singular in the sense of the endowments for utilities fixed, this means that in a neighborhood of $w$ there are economies with different number of equilibria.

\subsection{Examples of Economies that depend on the Initial Endowments}

As mentioned above, in the traditional sense the properties of the economies in a neighborhood of a singular economy depend on variable initial endowments while, as it is well known, utilities are fixed. This case is shown in an example where we consider $\alpha=8, w_{21}=r$, but we allow changes in $w_{12}$. Figure 3 shows this case, the singularities are economies such that $w_{12}=0.769$, or $w_{12}=0.7730$. In all neighborhoods of this points, we observe changes in the number of equilibria. Now allow us to modify the values of $\alpha$. As figure 3.a shows, the behavior of the economy changes. For values of $\alpha$ lower than 7.767, the possibility of changes in the number of equilibria disappear, and we obtain economies with uniqueness of equilibrium for all values of $w_{12}$, and then there are no singularities, nevertheless in a neighborhood of $\alpha=7.767$, and endowments given, we can observe big changes in the equilibrium set for small changes 
in utilities, for instance, for small changes in the values of $\alpha$. This situation is well known in economics.

Figure 3.a

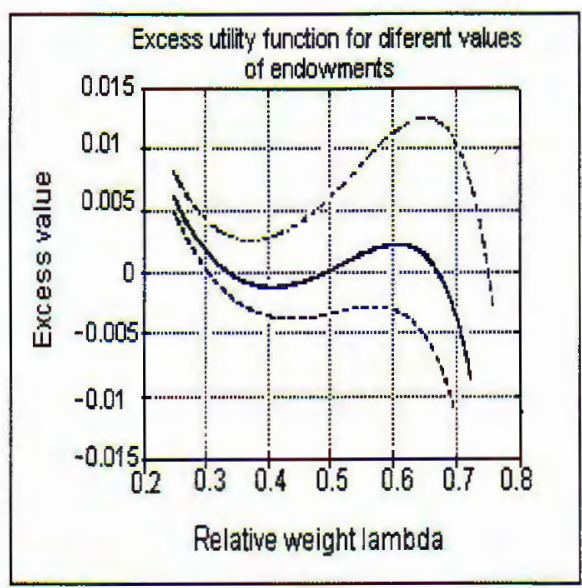

Figure 3.b

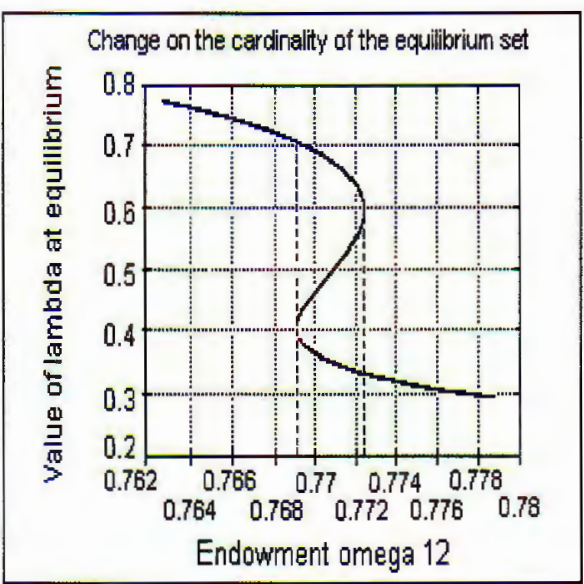

A well known situation, when a singular economy appears, changes in the endowments in a neighborhood of this singularity give rise to changes in the number of equilibria.

From the point of view of the singularity theory, we can consider the function $e$ as a function from the space formed by the cartesian product $\mathcal{U} \times \Omega \times \Delta$ in $\mathbb{R}^{n-1}$, and to consider changes in tastes or in endowment. An economy will be singular if it has critical points (singular Walrasian equilibria), i.e. if for some $\lambda \in \Delta, e_{u, w}(\vec{\lambda})=0$, and $\operatorname{rank} J e_{u, w}<n-1$. In a neighborhood of these economies, small changes in tastes or endowments may imply big changes in the behavior of the economy, for instance, changes in the number of Walrasian equilibria, and in its geometrical representation. Figure 4 shows different kinds of equilibrium sets for economies with different sets of utilities, this differences were obtained by changing the parameter $\alpha$. The doted curve shown in figure 4 represents the equilibrium set of an economy with a singular equilibrium, it separates regular economies with uniqueness of equilibrium for all values of the endowments, from economies such that the number of equilibria changes with the values of the endowments.

\section{Conclusions}

The existence of singularities is the support of the irreversibility. Small changes in endowments or tastes can imply big changes, and after these big changes turning back is only possible if the society makes concerted efforts.

The existence of singularities may be an answer to an ambitious question like: Why do crisis exist? If this result about the existence of singular economies could be a real possibility to explain crises, then a crisis would be the result of the structural conditions, and not a result of exogenous movements in fundamentals. We believe this to be the main motivation to analyze the structural 
characteristics of different kinds of singularities. Nevertheless, the knowledge of the kind of possible singularities in an economy allows us to characterize the kind of the unforseen future, wich in some sense means to have additional information.

Figure 4.

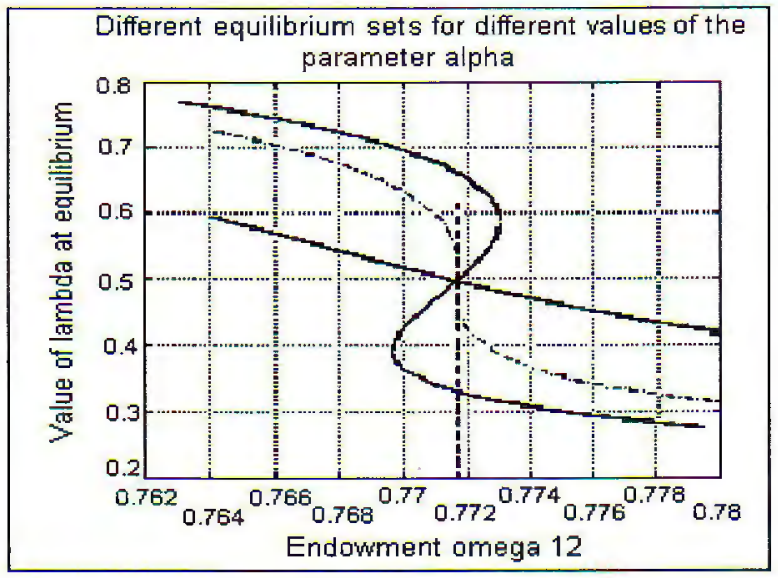

Variations in the values of the parameter $\alpha$ produce economies with qualitatively different equilibrium sets.

Economies with the same possible singularities will show a similar behavior. If there are no big changes in the neighborhood of regular economies, significative changes do occur in a neighborhood of a singularity, and this kind of changes are an important characterization of the economy.

Finally, we would like to bring into consideration the existing duality, between the change of tastes and the change in the endowments, as generators of steep changes in the economy. Singularities can appear from tastes or from the distribution of the initial resources of the society. In some cases, small mistakes in the measurement of the endowments, or in the tastes of the agents, can lead to unexpected, and irreversible results.

\section{Appendix A}

\section{A.1 Example of the Excess Utility Function}

Consider the economy $E=\left\{u_{\alpha, i}, w_{i}, \mathbb{R}_{+}^{l}, i=1,2\right\}$ whose utility functions are:

$$
\begin{gathered}
u_{\alpha, 1}=x_{11}-\frac{1}{\alpha} x_{12}^{-\alpha}, \\
u_{\alpha, 2}=-\frac{1}{\alpha} x_{21}^{-\alpha}+x_{22},
\end{gathered}
$$

and endowments $W=w_{1}+w_{2}$. Following the Negishi approach, we begin solving the optimization problem

$$
\text { Maximize } W_{\lambda}(x)=\lambda_{1} u_{1}\left(x_{1}\right)+\lambda_{2} u_{2}\left(x_{2}\right),
$$


restricted to the feasible set

$$
\mathcal{F}=\left\{x \in \mathbb{R}_{+}^{4}: \sum_{i=1}^{2} x_{i} \leq \sum_{i=1}^{2} w_{i}\right\} .
$$

Denoting $\lambda_{1}=1-\lambda$, it follows that $\lambda_{2}=\lambda$. We then write the excess utility function

$$
e_{u, w}=\left\{\begin{array}{l}
\left(\frac{1-\lambda}{\lambda}\right)^{\frac{\alpha}{1+\alpha}}-\left(\frac{1-\lambda}{\lambda}\right)^{\frac{1}{1+\alpha}}-w_{12}\left(\frac{1-\lambda}{\lambda}\right)+w_{21} \\
\left(\frac{1-\lambda}{\lambda}\right)^{\frac{-\alpha}{1+\alpha}}-\left(\frac{1-\lambda}{\lambda}\right)^{\frac{-1}{1+\alpha}}-w_{21}\left(\frac{1-\lambda}{\lambda}\right)^{-1}+w_{12}
\end{array} .\right.
$$

Then economies $E$, whose endowments are given by $w=\left(w_{11}, w_{12}, w_{21}, w_{22}\right)$ bearing

$$
W=\left(\begin{array}{l}
w_{11} \\
w_{12}
\end{array}\right)+\left(\begin{array}{l}
w_{21} \\
w_{22}
\end{array}\right)
$$

and when

$$
w_{12}=\frac{\alpha}{1+\alpha} \eta^{\frac{1}{1+\alpha}}-\frac{1}{1+\alpha} \eta^{\frac{\alpha}{1+\alpha}}
$$

are singular. Solving $e_{u}(\lambda, w)=0$, it is easy to see that in all neighborhoods of this economies, there exist economies with one equilibrium, and economies with three equilibria.

\section{A.2 A Degenerate Singular Economy}

Let $\eta=\lambda_{1} / \lambda_{2}$ be the relative social weight. Consider in (A.1.1) the excess utility function of the agent 1 ,

$$
e_{u_{1}, w_{1}}(\eta)=\eta^{\frac{\alpha}{1+\alpha}}-\eta^{\frac{1}{1+\alpha}}-w_{12} \eta+w_{21} .
$$

The first order derivative of this function with respect to $\eta$ is,

$$
\frac{\partial e_{u_{1}, w_{1}}(\eta)}{\partial \eta}=\frac{\eta^{-\frac{1}{1+\alpha}}}{1+\alpha}\left[\alpha-\eta^{\frac{1-\alpha}{1+\alpha}}\right]-w_{12}
$$

and the second derivative of $e_{u_{1}, w_{1}}$ respect to $\eta$ is equal to zero,

$$
\frac{\partial^{2} e_{u_{1}, w_{1}}(\eta)}{\partial \eta^{2}}=0
$$

if and only if $\eta=1$.

Substituting this value in the first derivative, we obtain that

$$
\frac{\partial e_{u_{1}, w_{1}}}{\partial \eta}(1)=0
$$


implying the equality

$$
\frac{\alpha-1}{1+\alpha}=w_{12} .
$$

Now consider an economy where $w_{12}=w_{21}$, it then follows that $e_{u_{1}, w_{1}}(1)=0$. So, if $\alpha=\left(1+w_{12}\right) /\left(1-w_{12}\right)$, and $w_{12}=w_{21}$, the excess utility function of the economy given in the example is a non Morse function, and then the economy

$$
E=\left\{u_{\alpha, 1}, u_{\alpha, 2}, w_{1}=\left(w_{11}, w_{12}\right), w_{2}=\left(w_{21}, w_{22}\right), w_{12}=w_{21}, \alpha=\frac{1+w_{12}}{1-w_{12}}\right\}
$$

is a degenerate singular economy, where $\lambda_{1}=\lambda_{2}=\frac{1}{2}$ is a singular degenerate equilibrium social weight.

\section{References}

Accinelli, E. (1996). Some Remarks on Uniqueness of Equilibrium in Economics with Infinitely Many Goods. Estudios Económicos, 6.

Accinelli, E. (1999). On Uniqueness of Equilibrium for Complete Markets with Infinitely Many Goods, and in Finance Models. Estudios de Economía, 26, pp. 45-61.

Accinelli, E., and M. Puchet (2001). A Characterization of Infinite Dimensional Walrasian Economies. Working Paper No. 7/701. Departamento de Economía, Facultad de Ciencias Sociales.

Balasko, Y. (1978). Economie et Théorie des Catastrophes. Mathématiques et Sciences Humaines, $16^{e}$ année, 64 .

Golubitsky, M., and V. Guillemin (1973). Stable Mappings, and their Singularities. Springer-Verlag. New-York-Hedelberg-Berlin.

Mas-Colell, A. (1985). General Equilibrium: A Differentiable Approach. Cambridge. Mas-Colell, A., M. Whinston, and J. Green (1995). Microeconomic Theory. Oxford.

Negishi, T. (1960). Welfare Economics, and Existence of an Equilibrium for a Competitive Economy, Metroeconómica 12.

Royden, H. (1963). Real Analysis. The Macmillan Company. 